made in the amplifiers between the microphones and the transmitting stations, and the range of audiofrequencies faithfully reproduced now extends up to 11,000 cycles per second. This should ensure that the quality of the broadcasting programmes is limited principally by the capabilities of the receiving instruments and the conditions under which they are used by listeners.

\section{Manufacture of Telephones in Sweden}

The trouble taken by large manufacturing firms abroad to acquaint foreigners with their products and the work they have done is worthy of imitation. During this year, the telephone factory of $I_{\text {. }}$. M. Ericsson of Stockholm has published two reviews written in excellent English and well illustrated, each giving about 70 pages of most readable matter mainly about automatic telephones and exchange stations. There is a description of a system which notifies electrically on panels in a bank the quotations from the stock exchange immediately they are fixed officially. These panels can be inspected by the public. The methods of protecting transmission lines from excess voltages due to atmospheric electricity by means of condensers are described and a full scientific description is given of their action. There being so many wooden buildings in Sweden, there is a great demand for automatic fire alarm systems. When a fire breaks out, the effects of the fire itself acting on the device at once summon the fire brigade. The new Ericsson bakelite telephones are described. A description is given of automatic exchanges in Iceland, Norway and Finland and there are many beautiful photographs. With the beginning of this year, the firm started publishing a series of highly technical papers on the theory of telephony and allied subjects. Of the four we have seen, one is in French and three are in English. They record much of the work carried out by the Research and Development Department of the Company.

\section{Railway Electrification}

IN the Electrical Supervisor, the journal of the Association of Supervising Electrical Engineers, of November, the presidential address of Mr. J. M. Kennedy to the Association is given. Mr. Kennedy makes useful suggestions on problems relating to the economic development and co-ordination of the electric supply industry. He points out that although railway electrification is a straight economic issue based on no increase of traffic, he considers it a much more productive line of capital development than road transport. As a comprehensive scheme for the whole of Great Britain, it is only a paying proposition at the expense of a reduction of personnel and of the total amount of coal used. He considers that both these disadvantages are certain to be outweighed by countervailing advantages. The electrification of railways will give traffic managers a new method of attracting traffic due to greater acceleration, speed, cleanliness and general comfort. In addition, the shorter trains run at more frequent intervals, the absence of smoke, and better time-keeping will help. Fxperience on the Southern Railway so far indicates that a very great increase in traffic is likely to result. A regular half-hourly service between London, Manchester and Birmingham would lead to a considerable increase in regular passenger traffic. Increased traffic will help to make good the apparent reduction in the number of employees, and the increase in electric production will also help. The electrification would not be completed for $15-20$ years and would therefore be assisting employment continuously during this period. The increase in the efficiency of transport will also add its share to reducing unemployment to its normal level.

\section{Gases in Metals}

$I_{T}$ is known that the presence of a minute trace of gas in a metal may greatly change its properties. For example, the magnetic permeability of commercially pure iron is greatly increased by eliminating the small amount of gas which it contains. In the Bell Laboratories Record of September, E. E. Schumacher gives an interesting account of the methods employed to free metals from gases, par. ticularly those used in the telephone industries. When the high degree of purity required for research purposes is desired, the metal is usually heated in a vacuum at a temperature above the melting point for a considerable time. But even at low pressures, sufficient gas may be left in the metal to be troublesome. When this occurs, alternately melting and partially solidifying the metal is employed in a high vacuum. In this way, almost complete elimination of the gas can be obtained. The metal to be freed of gas is placed in a shallow boat of fused aluminium oxide. This gives a large surface exposure and reduces the head of metal through which the gas must pass to escape. The apparatus is sealed in a pyrex glass tube connected to the pumping system. The tube is placed in a nichrome resistance furnace and a temperature of $450^{\circ} \mathrm{C}$. is maintained until gas is no longer liberated. A high frequency coil is then substituted for the nichrome furnace and the metal is melted by induced high-frequency current. It is possible to keep the metal at its melting temperature indefinitely without heating the pyrex glass tube to its melting point. The final pressure may be as low as one thousand millionth of an atmosphere. The comparison of the properties of the purified samples with those of samples of any given gas content is of great importance.

\section{Noiseless Underground Trains}

A serious drawback to underground trains is the noise in the carriages when the train is in motion. In many cases this makes conversation even between people sitting next to one another difficult, if not impossible. It is interesting therefore to hear that experiments are being carried out on one of the busiest of New York subways with the object of eliminating most of the noise nuisance. According to the Electrician of December 15, five cars equipped with special noise-control devices have been placed in service by the Inter-borough Rapid Transit Co. with the object of finding out how they attract the public. If the silent cars attract the passengers, the 